\title{
Performance Status Restriction in Phase III Cancer Clinical Trials
}

\author{
Joseph Abi Jaoude, MD1,*; Ramez Kouzy, MD1,*; Walker Mainwaring, BA2; Timothy A. Lin, MD³; Austin B. Miller, BS4; \\ Amit Jethanandani, MD, MPH${ }^{5}$; Andres F. Espinoza, MD²; Dario Pasalic, MD'; Vivek Verma, MD'; \\ Noam A. VanderWalde, MD ${ }^{6}$; Benjamin D. Smith, MD ${ }^{1}$; Grace L. Smith, MD, PhD, MPH ${ }^{1}$; C. David Fuller, MD, PhD'; \\ Prajnan Das, MD, MS, MPH${ }^{1}$; Bruce D. Minsky, MD'; Claus Rödel, MDD,8,9,10; Emmanouil Fokas, MD, DPhil 7,8,9,10; \\ Reshma Jagsi, MD, DPhil ${ }^{11}$; Charles R. Thomas Jr, MD ${ }^{12}$; Ishwaria M. Subbiah, MD'; \\ Cullen M. Taniguchi, MD, PhD ${ }^{1,+}$; and Ethan B. Ludmir, MD ${ }^{1,+}$
}

\section{ABSTRACT}

Background: Patients with good performance status (PS) tend to be favored in randomized clinical trials (RCTs), possibly limiting the generalizability of trial findings. We aimed to characterize trial-related factors associated with the use of PS eligibility criteria and analyze patient accrual breakdown by PS. Methods: Adult, therapeutic, multiarm phase III cancer-specific RCTs were identified through ClinicalTrials.gov. PS data were extracted from articles. Trials with a PS restriction ECOG score $\leq 1$ were identified. Factors associated with PS restriction were determined, and the use of PS restrictions was analyzed over time. Results: In total, 600 trials were included and 238,213 patients had PS data. Of those trials, 527 studies (87.8\%) specified a PS restriction cutoff, with 237 (39.5\%) having a strict inclusion criterion (ECOG PS $\leq 1$ ). Enrollment criteria restrictions based on PS (ECOG PS $\leq 1$ ) were more common among industry-supported trials $(P<.001)$ and lung cancer trials $(P<.001)$. Nearly half of trials that led to FDA approval included strict PS restrictions. Most patients enrolled across all trials had an ECOG PS of 0 to 1 (96.3\%). Even among trials that allowed patients with ECOG PS $\geq 2$, only $8.1 \%$ of those enrolled had a poor PS. Trials of lung, breast, gastrointestinal, and genitourinary cancers all included $<5 \%$ of patients with poor PS. Finally, only $4.7 \%$ of patients enrolled in trials that led to subsequent FDA approval had poor PS. Conclusions: Use of PS restrictions in oncologic RCTs is pervasive, and exceedingly few patients with poor PS are enrolled. The selective accrual of healthier patients has the potential to severely limit and bias trial results. Future trials should consider a wider cancer population with close toxicity monitoring to ensure the generalizability of results while maintaining patient safety.

J Natl Compr Canc Netw 2020;18(10):1322-1326 doi: $10.6004 /$ jnccn.2020.7578

\footnotetext{
${ }^{1}$ The University of Texas MD Anderson Cancer Center, Houston, Texas; ${ }^{2}$ Baylor College of Medicine, Houston, Texas; ${ }^{3}$ The Johns Hopkins University School of Medicine, Baltimore, Maryland; ${ }^{4}$ The University of Texas Health Science Center McGovern Medical School, Houston, Texas; ${ }^{5}$ The University of Tennessee Health Science Center College of Medicine, Memphis, Tennessee; ${ }^{6}$ West Cancer Center and Research Institute, Memphis, Tennessee; ${ }^{7}$ University of Frankfurt, Frankfurt, Germany; ${ }^{8}$ German Cancer Research Center, Heidelberg, Germany; ${ }^{9}$ German Cancer Consortium, Frankfurt, Germany; ${ }^{10}$ Frankfurt Cancer Institute, Frankfurt, Germany; ${ }^{11}$ University of Michigan, Ann Arbor, Michigan; and ${ }^{12}$ Oregon Health and Science University, Portland, Oregon.

*These authors contributed equally and are co-first authors.
}

\footnotetext{
†These authors contributed equally as senior authors
}

\section{Background}

Performance status (PS) is an essential metric in cancer care $^{1-3}$ PS scores are based on estimations of the patient's ability to perform activities of daily living and are commonly used to predict whether a patient can tolerate and respond to therapy. ${ }^{4}$ The most common adult PS scales in clinical practice are the ECOG PS and the Karnofsky performance status (KPS) scores. ${ }^{5,6}$ ECOG scores range from 0 (full ambulation without symptoms) to 5 (dead) and KPS scores range from 100 (full normal functioning) to 0 (dead). ${ }^{7-9}$

Patients with ECOG PS 0 to 1 are broadly considered to have a good PS and tend to have better clinical and survival outcomes. ${ }^{2,10,11}$ Such patients have been historically favored in clinical trials, which may influence trial results and subsequent FDA drug approvals. ${ }^{12}$ Similar practices may have important implications for the generalizability of trial findings, because a considerable proportion of patients with cancer do not present at diagnosis with good PS, which portends poorer survival., ${ }^{8,9,13-15}$ A striking example is lung cancer, in that more than one-third of patients with lung cancer present with poor PS (ECOG PS $\geq 2$ ). ${ }^{13}$

To understand the landscape of PS restriction, we analyzed a large collection of phase III cancer randomized clinical trials (RCTs). We identified trial-related factors associated with the use of PS-restrictive eligibility criteria (defined as the inclusion of only patients with ECOG PS $\leq 1$ ) and analyzed the use of strict PS eligibility criteria over time. We further determined the actual patient accrual by PS across all trials.

\section{Methods}

\section{Study Design and Data Sources}

A database query was performed through ClinicalTrials. gov to search for oncologic phase III RCTs as described in previous research. ${ }^{16}$ The following advanced search parameters were used: other terms: "cancer"; study type:

See page 1431 for related commentary. 
"all studies"; status: excluded "not yet recruiting"; phase: phase III; study results: "with results." This search yielded 1,239 trials. Trials were subsequently screened for therapeutic, cancer-specific, phase III, randomized, multiarm trials in adult patients. Figure 1 presents the trial screening and eligibility criteria adopted in this study based on the PRISMA methodology. ${ }^{17}$

Eligibility criteria for each trial were assessed through data provided by ClinicalTrials.gov along with the trial protocol and primary publication of trial results, if available. Trial screening and parameter identification were independently performed by 2 individuals. No Institutional Review Board approval was required because all data were based on publicly available triallevel data without use of protected health information; no informed consent was required.

\section{Statistical Analysis}

Data regarding the use of inclusion or exclusion criteria based on patients' PS were collected from ClinicalTrials. gov, the primary endpoint article publication, or attached protocols. Data regarding patient accrual breakdown by PS were obtained from primary publication of trial primary endpoint results. All PS results were coded and analyzed using the ECOG measure, because most trials included used the ECOG PS system; KPS-based data were converted to ECOG PS using previously described methods. ${ }^{7}$

Any trial that specified an upper PS restriction cutoff of an ECOG PS $\leq 1$ was considered to have a strict PS exclusion criterion. Differences in the proportions of trials that had a strict exclusion criterion (ECOG $\leq 1$ ) across groups were assessed using Pearson chi-square tests. Binary logistic regression was used for analysis of temporal changes in strict PS-restrictive eligibility criteria utilization. Statistical significance was set a priori at a 2 -sided $\alpha$ of .05. All analyses were performed using SPSS Statistics, version 24 (IBM Corp).

\section{Results}

\section{PS Restriction Criteria}

A total of 600 trials were included in our study, comprising 428,560 accrued patients (Figure 1). Most trials $(\mathrm{n}=527 ; 87.8 \%)$ specified an upper PS restriction cutoff as part of their enrollment criteria, with 237 studies (39.5\%) having a strict inclusion criterion $(\mathrm{ECOG} \leq 1)$.

Table 1 highlights specific trial factors associated with the use of strict PS inclusion criteria. Industryfunded trials had higher rates of PS-restrictive criteria compared with non-industry-supported trials $(43.5 \%$ vs $25.6 \%$, respectively; $P<.001$ ). Meanwhile, cooperative group trials had fewer PS restrictions than noncooperative group-sponsored trials $(30.3 \%$ vs $43.7 \%$, respectively; $P=.002$ ). When analyzing trials by disease

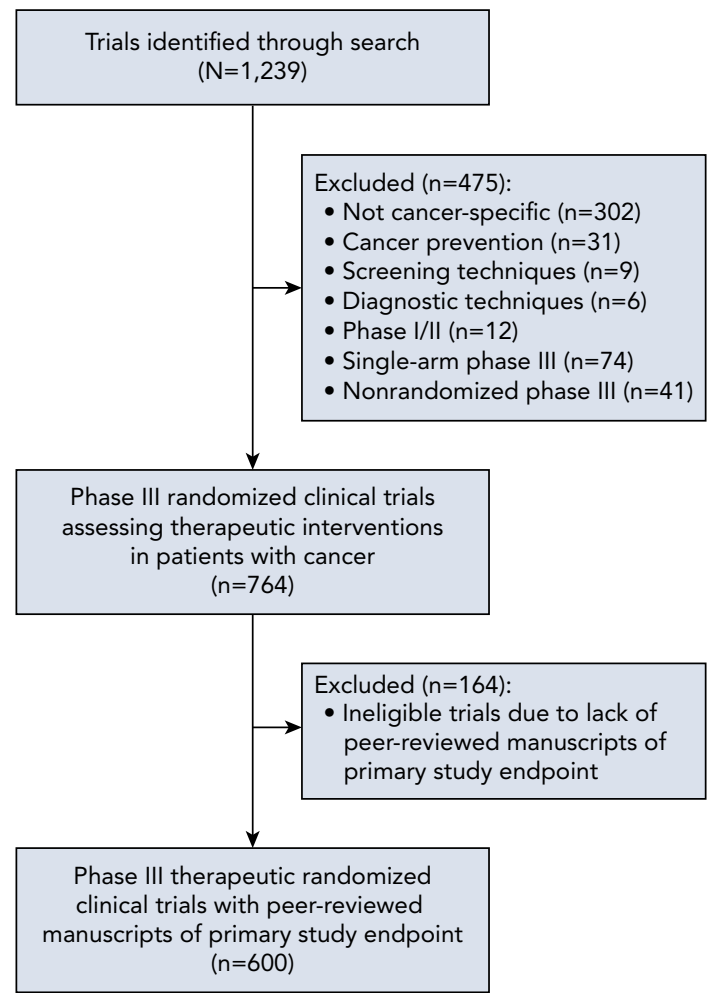

Figure 1. Flowchart of clinical trial screening, eligibility, and inclusion. Abbreviation: RCTs, randomized clinical trials.

site, we found that lung cancer trials had the highest rates of strict PS restrictions (57.3\%), and hematologic cancer trials had the lowest $(16.8 \% ; P<.001)$. Systemic therapy trials had the highest PS restrictions (45.1\%; $P<.001)$. Furthermore, the use of strict PS eligibility criteria did not differ between trials that met or did not meet their primary endpoint $(P=.61)$. Finally, nearly half of trials $(45.5 \%)$ that subsequently led to FDA drug approval had a strict PS inclusion criterion (Table 1).

Examining the use of strict PS eligibility criteria over time revealed that $37.2 \%$ of trials published between 2007 and 2012 and $40.4 \%$ of trials published between 2013 and 2018 were PS-restrictive. Binary logistic regression revealed the stable use of strict PS eligibility criteria over time (odds ratio, $1.01 ; P=.79$ ).

\section{Patient Accrual Breakdown by PS}

Of the 600 trials included, $443(73.8 \%)$ included data on patient PS. Specifically, 238,213 (55.6\%) of the 428,560 patients accrued across all included trials had PS data reported in the main article. Table 2 presents the PS distribution of accrued patients across different trials. Most patients included in cancer clinical trials had an ECOG PS of either $0(n=133,982 ; 56.2 \%)$ or $1(n=95,547$; $40.1 \%$ ), with only $3.6 \%$ of patients having an ECOG PS of $\geq 2$. Critically, even among the 363 trials that allowed 


\begin{tabular}{|c|c|c|}
\hline $\begin{array}{r}\text { Tria } \\
\text { Ex }\end{array}$ & $\begin{array}{l}\text { With Restrictive } \\
\text { usion Criteria of } \\
\text { ECOG PS } \leq 1 \\
\text { n (\%) }\end{array}$ & $P$ Value ${ }^{\mathrm{a}}$ \\
\hline Industry funding of trial ${ }^{b}$ & & $<.001$ \\
\hline Yes & $03 / 467(43.5)$ & \\
\hline No & 34/133 (25.6) & \\
\hline Cooperative group trial ${ }^{b}$ & & .002 \\
\hline Yes & $57 / 188(30.3)$ & \\
\hline No & 180/412 (43.7) & \\
\hline Disease site ${ }^{c}$ & & $<.001$ \\
\hline Breast & 46/105 (43.8) & \\
\hline Gastrointestinal & 36/76 (47.4) & \\
\hline Genitourinary & $35 / 70(50.0)$ & \\
\hline Head and neck & 9/22 (40.9) & \\
\hline Hematologic & 20/119 (16.8) & \\
\hline Lung & $51 / 89(57.3)$ & \\
\hline Treatment modality ${ }^{d}$ & & $<.001$ \\
\hline Systemic therapye & $210 / 466(45.1)$ & \\
\hline Radiotherapy & $4 / 14(28.6)$ & \\
\hline Surgery & 1/7 (14.3) & \\
\hline Supportive care $f$ & $21 / 110(19.2)$ & \\
\hline Trial success (primary endpoint met) & & .61 \\
\hline Yes & 118/306 (38.6) & \\
\hline No & 119/294 (40.5) & \\
\hline Subsequent FDA approvalg & & .074 \\
\hline Yes & 71/156 (45.5) & \\
\hline No & 166/444 (37.4) & \\
\hline
\end{tabular}

Abbreviation: PS, performance status.

aBased on chi-square test.

bIndustry funding and cooperative group sponsorship were considered independent variables, because some trials were both industry-funded and performed through a cooperative group.

cAnalysis by disease site was limited to studies with a defined single disease site. 'Modality addressed the primary intervention as part of the randomization. eSystemic therapy trials, including chemotherapy, targeted systemic agents, and immunotherapy, accounted for most trials by modality; they used systemic therapies to improve disease-related outcomes (eg, overall survival, diseasefree survival).

fSupportive care trials were those in which the intervention aimed to reduce disease- or treatment-related toxic effects as the primary endpoint.

9The trials led to subsequent FDA approval of the drug being tested.

patients with poor PS to enroll (ECOG PS $\geq 2$ ), a sizable majority of accrued patients had ECOG PS 0 or 1 $(\mathrm{n}=95,786 ; 91.9 \%)$. As such, despite those trials allowing the enrollment of patients with poor PS, only $8.1 \%$ of enrolled patients had an ECOG PS $\geq 2$.

When examining specific trial factors, we found that that $>90 \%$ of accrued patients had good PS (ECOG $\leq 1$ ), irrespective of trial factors and initial eligibility criteria. Hematologic cancer trials had the largest proportion of patients with ECOG PS $\geq 2$ (8.7\%), followed by head and neck cancer trials $(7.4 \%)$, whereas lung, breast, gastrointestinal, and genitourinary cancer trials included $<5 \%$ of patients with poor PS $(P<.001$; Table 2$)$. Trials that met their primary endpoint included only $3.6 \%$ of patients with poor PS. Finally, only $4.7 \%$ of patients who were enrolled in trials that led to subsequent FDA drug approval had a poor PS (ECOG PS $\geq 2$ ).

\section{Discussion}

In this evaluation of cancer RCTs, a large proportion of trials $(40 \%)$ had strict PS eligibility criteria, excluding patients with ECOG PS $\geq 2$. Our data showed that the use of strict PS exclusion criteria did not improve over time, despite calls for reevaluating clinical trial eligibility criteria. ${ }^{12}$ The pervasiveness of strict trial eligibility criteria can lead to the underrepresentation of patients with poor PS in clinical trials. In that context, previously published data showed similar trends in the underrepresentation of older patients, female patients, and $\mathrm{racial} / \mathrm{ethnic} \mathrm{mi}$ nority patients. ${ }^{18}$ As such, the generalizability of phase III trial results is called into question.

A possible rationale behind the use of stricter PS eligibility criteria might be to ensure patient safety and treatment tolerance. ${ }^{19}$ However, recent data from patients receiving novel therapies, including immune checkpoint inhibitors, suggest similar toxicity profiles between patients with good and poor PS. ${ }^{20}$ Moreover, the scoring of PS is often subjective and dependent on physician interpretation of patients' well-being. ${ }^{8,9}$ PS measures are mainly used to assess whether a patient would tolerate the use of systemic therapy, but they are also subjective regarding physician interpretation of the patient's execution of activities of daily living (with emphasis on ambulatory status). ${ }^{4}$ As such, although some PS restrictions may be justified, the fundamentally subjective nature of PS may affect physician reporting based on protocol requirements. Thus, the inclusion and close monitoring of patients with poor PS, as an alternative to exclusion, could be considered in cancer clinical trials. In addition, including patients with poor PS is important to consider given that exclusion of these patients may impact study results because healthier patients tend to have better prognoses. ${ }^{1-3}$

In many malignancies, poor PS is often a risk factor for early mortality, which may in turn reduce the effect size of the intervention, hence leading trials to not meet their primary endpoint. ${ }^{10,11,21}$ Inclusion of patients with poor PS and a high risk for mortality may bias results, and trials may be underpowered and fail to show a true effect even when one exists. Increasing the heterogeneity of patients by PS within the trial population is therefore not without risk. Furthermore, both the absolute and relative effects of treatment may differentially impact patients by PS, which may contribute 


\begin{tabular}{|c|c|c|c|}
\hline Trial Factor & $\begin{array}{c}\text { ECOG PS 0-1 } \\
n(\%)\end{array}$ & $\begin{array}{c}\text { ECOG PS 2-4 } \\
n(\%)\end{array}$ & $P$ Value ${ }^{a}$ \\
\hline All trials & $229,529(96.4)$ & $8,684(3.6)$ & \\
\hline \multicolumn{2}{|c|}{ Restrictive PS eligibility (ECOG PS 0-1) } & & $<.001$ \\
\hline Yes & $133,743(99.9)$ & $188(0.1)$ & \\
\hline No & $95,786(91.9)$ & $8,496(8.1)$ & \\
\hline \multicolumn{2}{|c|}{ Industry funding of trial ${ }^{b}$} & & $<.001$ \\
\hline Yes & $204,265(96.2)$ & $8,144(3.8)$ & \\
\hline No & $25,264(97.9)$ & $540(2.1)$ & \\
\hline \multicolumn{2}{|c|}{ Cooperative group trial ${ }^{b}$} & & $<.001$ \\
\hline Yes & $53,913(97.2)$ & $1,569(2.8)$ & \\
\hline No & $174,174(96.1)$ & $7,108(3.9)$ & \\
\hline \multicolumn{2}{|l|}{${\text { Disease } \text { site }^{c}}$} & & $<.001$ \\
\hline Breast & $46,056(98.9)$ & $530(1.1)$ & \\
\hline Gastrointestinal & $43,933(97.1)$ & $1,330(2.9)$ & \\
\hline Genitourinary & $34,553(97.5)$ & $901(2.5)$ & \\
\hline Head and neck & $4,697(92.6)$ & $378(7.4)$ & \\
\hline Hematologic & $23,391(91.3)$ & $2,241(8.7)$ & \\
\hline Lung & $45,852(95.2)$ & $2,330(4.8)$ & \\
\hline \multicolumn{2}{|l|}{ Treatment modality ${ }^{d}$} & & $<.001$ \\
\hline Systemic therapy ${ }^{\mathrm{e}}$ & $199,499(96.5)$ & $7,222(3.5)$ & \\
\hline Radiotherapy & 4,877 (99.3) & $33(0.7)$ & \\
\hline Surgery & $285(96.3)$ & $11(3.7)$ & \\
\hline Supportive care ${ }^{f}$ & $22,981(94.8)$ & $1,268(5.2)$ & \\
\hline \multicolumn{3}{|c|}{ Trial success (primary endpoint met) } & .019 \\
\hline Yes & $111,118(96.4)$ & $4,093(3.6)$ & \\
\hline No & $118,411(96.3)$ & $4,591(3.7)$ & \\
\hline \multicolumn{2}{|c|}{ Subsequent FDA approval9 } & & $<.001$ \\
\hline Yes & $67,468(95.3)$ & $3,361(4.7)$ & \\
\hline No & $162,061(96.8)$ & $5,323(3.2)$ & \\
\hline
\end{tabular}

Abbreviation: PS, performance status.

abased on chi-square test.

bIndustry funding and cooperative group sponsorship were considered independent variables because some trials were both industry-funded and performed through a cooperative group.

'Analysis by disease site was limited to studies with a defined single disease site.

${ }^{\mathrm{d}}$ Modality addressed the primary intervention as part of the randomization. eSystemic therapy trials, including chemotherapy, targeted systemic agents, and immunotherapy, accounted for most trials by modality; they used systemic therapies to improve disease-related outcomes (eg, overall survival, diseasefree survival).

fSupportive care trials were those in which the intervention aimed to reduce disease- or treatment-related toxic effects as the primary endpoint.

9The trials led to subsequent FDA approval of the drug being tested.

to heterogeneity if a wider spectrum of patients by PS are enrolled. On the other hand, differential effects of treatment across the PS gradient must be captured; if the relative benefit of an intervention is higher among patients with poor PS, for instance, then the imperative to ensure that patients with poor PS are adequately represented among trial participants is all the stronger. A potential solution to this issue is a trial design that stratifies patients by PS in order to ensure sufficient power across the PS range to measure effects in patients with both good and poor PS. Another option is to perform trials targeted for patients with poor PS only, in which special care and monitoring would be implemented to maintain patient safety. Along this line, 2 ongoing phase II lung cancer trials are focusing on patients with poor PS only (ClinicalTrials.gov identifiers: NCT00193336 and NCT00892710). Such trials would be analogous to studies that have restricted their inclusion criteria to older patients (ie, limiting trials to only patients aged $\geq 65$ years). ${ }^{22}$ Furthermore, studies analyzing targeted therapy rather than cytotoxic chemotherapy may be particularly relevant in patients with poor PS, as those patients may be particularly susceptible to chemotherapy-induced toxicity (NCT02733159). ${ }^{20}$

When we examined actual patient accrual breakdown, $96 \%$ of patients accrued to trials had good PS (ECOG PS $\leq 1)$. Most patients $(>90 \%)$ across trials had good PS irrespective of industry sponsorship of the trial, disease site, treatment modality, and more. Notably, low accrual of patients with poor PS seems to occur via a 2-pronged approach. The first centers on the use of PSrestrictive eligibility criteria-only $0.1 \%$ of patients enrolled in PS-restrictive trials had poor PS. The second is seemingly independent of the first; that is, even among trials in which accrual of patients with poor PS was permitted, it occurred at low rates-only $8.1 \%$ of enrolled patients among PS-permissive trials had an ECOG PS of 2 to 4 . The low enrollment of patients with poor PS even among PS-permissive trials highlights the intrinsic selection for healthy patients as trial participants and represents an additional challenge beyond specific eligibility criteria in ensuring that trial participants and results are generalizable to the realworld population. Along these lines concerning risks of limited generalizability, trials that led to subsequent FDA drug approval included $<5 \%$ of patients with poor PS. Notably, lung cancer trials also included $<5 \%$ of patients with poor PS, even though approximately one-third of patients with lung cancer present with ECOG PS $\geq 2 .{ }^{13,14}$ Although physician selection bias may play a role in the underrepresentation of patients with poor PS in trials, another possible explanation may be that patients with poor PS have less willingness to participate in RCTs because of their multiple comorbidities that may affect quality of life, making them reluctant to add new treatments. In addition, preexisting financial toxicity for patients with comorbidities or poor PS may play a role in limiting access to RCTs. ${ }^{23}$ 
Our study has some limitations worth noting. First, only 1 clinical trial registry based in the United States was screened, and therefore our findings may not apply to cancer trials accruing outside of the United States. Second, not all trial patients had PS information, and therefore selective reporting may affect the true profile of disparities by PS among trial participants. Nevertheless, although previous studies analyzed population discrepancies in clinical trials (age, sex, race), our study is the first to explore PS restrictions in cancer trials and quantify the absolute proportions of patients by PS accrued across trials. ${ }^{16,18}$

\section{Conclusions}

Use of strict PS restrictions in phase III cancer RCTs is pervasive. Moreover, the low accrual of patients with poor PS is strikingly common among cancer trials, irrespective of PS-specific trial eligibility criteria. The selective inclusion of healthy patients may severely limit the generalizability of trial results. Future trials should consider a more representative population with cancer, including more patients with poor PS who undergo close toxicity monitoring, to ensure better generalizability of results while maintaining patient safety.

\section{Acknowledgments}

We thank all of the patients who participated in the clinical trials analyzed.

Submitted March 7, 2020; accepted for publication April 16, 2020

Author contributions: Study concept and design: Jaoude, Kouzy, Taniguchi, Ludmir. Data collection: Jaoude, Kouzy, Mainwaring, Lin, Miller, Jethanandani, Espinoza, Ludmir. Data analysis and interpretation: All authors. Manuscript preparation: All authors.

Disclosures: Dr. VanderWalde has disclosed that he receives consulting fees from Vector Oncology. Dr. B.D. Smith has disclosed that he has received grant/ research support from Varian Medical Systems, and has an equity interest in Oncora Medical. Dr. Fuller has disclosed that he receives royalty income from Demos Medical Publishing, and honoraria from Elekta AB. Dr. Das has disclosed that he receives consulting fees from Adlai Nortye. Dr. Jagsi has disclosed that she has is a scientific advisor for Equity Quotient, has received honoraria from Amgen and Vizient, and has received grant/research support from the Doris Duke Foundation, the Greenwall Foundation, the Komen Foundation, and Blue Cross Blue Shield of Michigan. The remaining authors have disclosed that they have not received any financial consideration from any person or organization to support the preparation, analysis, results, or discussion of this article.

Funding: Dr. Taniguchi is supported by funding from NIH (award R01CA227517-01A1), Cancer Prevention \& Research Institute of Texas (CPRIT; grant RR140012), V Foundation (V2015-22), the Kimmel Foundation, Sabin Family Foundation Fellowship, and the McNair Foundation. This manuscript is also supported by NIH P30 CA016672.

Correspondence: Cullen M. Taniguchi, MD, PhD, Department of Radiation Oncology, The University of Texas MD Anderson Cancer Center, 1515 Holcombe Boulevard, Unit 1050, Houston, TX 77030

Email: ctaniguchi@mdanderson.org; and

Ethan B. Ludmir, MD, Department of Radiation Oncology, The University of Texas MD Anderson Cancer Center, 1400 Pressler Street, Unit 1422, Houston, TX 77030. Email: ebludmir@mdanderson.org

\section{References}

1. Gronlund B, Høgdall C, Hansen $\mathrm{HH}$, et al. Performance status rather than age is the key prognostic factor in second-line treatment of elderly patients with epithelial ovarian carcinoma. Cancer 2002;94:1961-1967.

2. Jang RW, Caraiscos VB, Swami N, et al. Simple prognostic model for patients with advanced cancer based on performance status. J Oncol Pract 2014;10:e335-341.

3. Kocher HM, Patel S, Linklater K, et al. Increase in the incidence of oesophagogastric carcinoma in the South Thames region: an epidemiological study. Br J Surg 2000;87:362-373.

4. Prigerson HG, Bao Y, Shah MA, et al. Chemotherapy use, performance status, and quality of life at the end of life. JAMA Oncol 2015;1:778-784.

5. Oken MM, Creech RH, Tormey DC, et al. Toxicity and response criteria of the Eastern Cooperative Oncology Group. Am J Clin Oncol 1982;5: 649-655

6. Karnofsky DA, Burchenal JH. The clinical evaluation of chemotherapeutic agents in cancer. In: MacLeod CM, ed. Evaluation of Chemotherapeutic Agents. New York, NY: Columbia University Press; 1949:191-205.

7. West $\mathrm{H}$, Jin J. Performance status in patients with cancer. JAMA Oncol 2015;1:998.

8. Leal AD, Allmer C, Maurer MJ, et al. Variability of performance status assessment between patients with hematologic malignancies and their physicians. Leuk Lymphoma 2018;59:695-701.

9. Sørensen JB, Klee M, Palshof T, et al. Performance status assessment in cancer patients. An inter-observer variability study. Br J Cancer 1993;67: 773-775.

10. Capewell S, Sudlow MF. Performance and prognosis in patients with lung cancer. Thorax 1990;45:951-956.

11. Salloum RG, Smith TJ, Jensen GA, et al. Survival among non-small cell lung cancer patients with poor performance status after first line chemotherapy. Lung Cancer 2012;77:545-549.

12. Jin S, Pazdur R, Sridhara R. Re-evaluating eligibility criteria for oncology clinical trials: analysis of investigational new drug applications in 2015 J Clin Oncol 2017;35:3745-3752.
13. Lilenbaum RC, Cashy J, Hensing TA, et al. Prevalence of poor performance status in lung cancer patients: implications for research. J Thorac Oncol 2008;3:125-129.

14. Buccheri G, Ferrigno D, Tamburini M. Karnofsky and ECOG performance status scoring in lung cancer: a prospective, longitudinal study of 536 patients from a single institution. Eur J Cancer 1996;32:1135-1141.

15. Corrêa GTB, Bandeira GA, Cavalcanti BG, et al. Analysis of ECOG performance status in head and neck squamous cell carcinoma patients: association with sociodemographical and clinical factors, and overall survival. Support Care Cancer 2012;20:2679-2685.

16. Ludmir EB, Mainwaring W, Lin TA, et al. Factors associated with age disparities among cancer clinical trial participants. JAMA Oncol 2019;5:1769-1773.

17. Liberati A, Altman DG, Tetzlaff J, et al. The PRISMA statement for reporting systematic reviews and meta-analyses of studies that evaluate healthcare interventions: explanation and elaboration. BMJ 2009;339:b2700

18. Murthy $\mathrm{VH}$, Krumholz HM, Gross CP. Participation in cancer clinical trials: race-, sex-, and age-based disparities. JAMA 2004;291:2720-2726.

19. George SL. Reducing patient eligibility criteria in cancer clinical trials. J Clin Oncol 1996;14:1364-1370.

20. Shah NJ, Blackburn M, Cook MR, et al. Real-world outcomes of underrepresented patient populations treated with immune checkpoint inhibitors (ICls): African American descent, poor ECOG performance status, and chronic viral infections [abstract]. J Clin Oncol 2019; 37(Suppl):Abstract 2587.

21. Hoang $T, X u$ R, Schiller JH, et al. Clinical model to predict survival in chemonaive patients with advanced non-small-cell lung cancer treated with third-generation chemotherapy regimens based on Eastern Cooperative Oncology Group data. J Clin Oncol 2005;23:175-183.

22. Perry JR, Laperriere N, O'Callaghan CJ, et al. Short-course radiation plus temozolomide in elderly patients with glioblastoma. N Engl J Med 2017 376:1027-1037

23. Chino F, Zafar SY. Financial toxicity and equitable access to clinical trials Am Soc Clin Oncol Educ Book 2019;39:11-18. 